\title{
Influência da ooforectomia e da gravidez na função fagocitária do sistema mononuclear fagocitário em modelo experimental
}

\author{
The influence of oophorectomy and pregnancy on the phagocytic function of the \\ phagocytic mononuclear system in experimental model
}

Leonardo de Souza Vasconcellos ${ }^{1}$, Kelly Renata Sabino², Andy Petroianu ${ }^{3}$

\begin{abstract}
unitermos resumo
Ooforectomia

Introdução: A interação dos hormônios ovarianos e placentários com o sistema imune ainda é controversa

Gravidez na literatura. Objetivos: Determinar a influência da ooforectomia e da gravidez na atividade fagocitária do sistema mononuclear fagocitário (SMF) em modelo experimental. Material e método: Foram utilizadas Sistema mononuclear fagocitário 45 ratas wistar adultas divididas em três grupos $(n=15)$ : grupo 1 - controle; grupo 2 - ratas ooforecto-

${ }^{99} \mathrm{mTc}$ enxofre coloidal mizadas; grupo 3 - ratas grávidas. A função do sistema mononuclear fagocitário foi determinada pela captação de enxofre coloidal marcado com $99 \mathrm{mT}$ c pelos órgãos do SMF, além de avaliar a quantidade de radiofármaco remanescente na circulação sangüínea por meio de um coágulo sangüíneo. O peso e a radiação de cada amostra foram medidos e comparados pelo teste $t$ de Student. Realizou-se também análise histológica desses órgãos. Resultados: Em todos os animais, a captação do radiofármaco foi maior no fígado, seguido por baço e pulmão. O coágulo sangüíneo apresentou uma quantidade mínima de radiação. A ooforectomia não foi acompanhada de mudanças na captação do colóide. Entretanto as ratas grávidas registraram menor captação do colóide no fígado e maior captação no pulmão. Não houve alteração na histoarquitetura dos órgãos estudados. Conclusões: Conclui-se que a gravidez altera a atividade fagocitária do SMF, porém a ooforectomia não parece interferir nessa função.
\end{abstract}

\section{abstract}

Introduction: The influence of ovarian and placental hormones on the immune system is still controverse in the literature. Objectives: Assess the influence of oophorectomy and pregnancy on the phagocytic function of phagocytic mononuclear system (PMS) in experimental model. Material and Method: 45 adults female wistar rats were divided into three groups ( $\mathrm{n}=15$ ): Group 1 - control, Group 2 -oophorectomized rats, Group 3-pregnant rats. The phagocytic function of PMS was assessed by uptake of sulphur colloid labeled with ${ }^{99} \mathrm{mTc}$ by liver, spleen and lung. The remnant colloid in the blood steam was verified by mears of a blood clot. The weight and radioactive level of the samples were measured and the results were compared by Student's $t$ test, with significance for $p<0.05$. Histological analyses of these organs were also performed. Results: The scintigraphic values were higher in liver followed by spleen and lung. The blood clot presented only little amount of radiation. The oophorectomized rats did not registered alterations in colloids uptake when compared with the control group. Although the pregnant rats registered lower radiation in the liver and higher in the lung no histological abnormality was found in all groups. Conclusions: In conclusion, pregnancy interferes with the phagocytic function of PMS, however oophorectomy does not seem to modify this function.

1. Professor substituto do Departamento de Propedêutica Complementar da Faculdade de Medicina da Universidade Federal de Minas Cerais (FM/UFMG); médico patologista clínico; mestrando em cirurgia pela FM/UFMG.

2. Cirurgiã-geral; residente de Ginecologia e Obstetrícia do Hospital Covernador Israel Pinheiro, em Belo Horizonte.

3. Professor-titular do Departamento de Cirurgia da Faculdade de Medicina da UFMG; livre-docente da Faculdade de Medicina de Ribeirão Preto da Universidade de São Paulo (FMRP/USP); livredocente da Escola Paulista de Medicina da Universidade Federal de São Paulo (EPM/UNIFESP); doutor em Fisiologia e Farmacologia pelo Instituto de Ciências Biológicas (ICB) da UFMG; pesquisador IA do Conselho Nacional de Desenvolvimento Científico e Tecnológico (CNPq). 


\section{Introdução}

O sistema mononuclear fagocitário (SMF) responsabiliza-se por grande parte da imunidade celular, atuando na defesa contra microorganismos, incluindo bactérias, fungos, vírus, parasitas e corpos estranhos, bem como na remoção de células mortas, partículas inaladas, entre outros. Suas células apresentam motilidade e respondem a fatores quimiotáxicos derivados principalmente de linfócitos $T^{(44,32)}$.

As células do SMF são produzidas na medula óssea e lançadas na corrente sangüínea sob a forma de monócitos para posteriormente migrarem aos tecidos, na forma de macrófagos ou histiócitos, e se estabelecerem em diversos tecidos ${ }^{(52)}$. O SMF também atua na modulação do sistema imune humoral, controla a hematopoiese, além de produzir enzimas, componentes do sistema complemento, proteínas de ligação, citocinas, fatores de coagulação e de angiogênese, bem como fatores de crescimento ${ }^{(20)}$.

O metabolismo dos esteróides sexuais femininos vem sendo pesquisado na literatura, porém, devido à sua complexidade, ainda não há consenso sobre a totalidade de suas funções, sendo necessários maiores esclarecimentos sobre as diversas interações endócrinas com o metabolismo corpóreo. A privação dos hormônios ovarianos acarreta distúrbios endócrinos e funcionais, como disfunção sexual, perda da libido, maior risco de osteoporose e de doenças cardíacas, níveis alterados de lipoproteínas, entre outros ${ }^{(48)}$. Do mesmo modo, durante a gravidez o organismo materno também sofre diversas adaptações anatômicas, fisiológicas, bioquímicas e psíquicas. Muitas dessas alterações já foram estudadas na literatura, como náuseas, vômitos, aumento do apetite, ganho ponderal, aumento dos fatores de coagulação, além de diminuição da albumina, aumento da fosfatase alcalina, elevação da volemia, aumento da freqüência cardíaca, hipocloridria, hipercolesterolemia, redução do peristaltismo, etc. ${ }^{(47)}$. Contudo são conflitantes as informações disponíveis sobre os efeitos da ooforectomia e da gravidez no sistema de defesa.

Os níveis de imunoglobulinas séricas (IgG, $\lg \mathrm{A}, \lg \mathrm{M}$, $\lg D$ e $\lg \mathrm{E})$ e o de células linfocitárias do tipo $B$ são mantidos em níveis normais durante a gravidez, segundo a maioria dos autores ${ }^{(24,36)}$. Por outro lado, diversos trabalhos identificaram uma queda sérica de anticorpos humorais, provavelmente por efeito hemodilucional decorrente do aumento da volemia no período gestacional ${ }^{(19,22)}$. Quanto à imunidade celular, é conhecida a leucocitose (14.000 a 18.000 céls $/ \mathrm{mm}^{3}$ ), que se torna mais pronunciada no período do trabalho de parto. Ela é principalmente constituída por polimorfonucleares, com diferencial variável de basófilos e eosinófilos ${ }^{(9,47)}$.

Além das alterações quantitativas, as variações funcionais das células imunitárias também já foram foco de estudo. Krause et al. (1987) observaram diminuição da quimiotaxia e da atividade fagocitária entre o segundo trimestre do período gestacional e o primeiro trimestre de puerpério ${ }^{(24,26,50)}$. Outros estudos relacionaram a depressão imunitária gestacional com a melhora clínica de doenças auto-imunes ${ }^{(1,2,49)}$.

Na década de 1980, alguns trabalhos postulavam que o sistema imune pode ser regulado pelos esteróides sexuais, por interações no eixo hipotalâmico-hipofisáriogonadal-tímico ${ }^{(15,25,35)}$. Posteriormente, essa teoria passou a ser subsidiada pela possibilidade de hormônios gonadais modularem a resposta imune e contribuírem para o tratamento de doenças auto-imunes. Por outro lado, a redução dos hormônios sexuais diminui a função dos macrófagos e aumenta a suscetibilidade para infecções ${ }^{(23)}$.

As enzimas antioxidantes dos macrófagos, a catalase e a superóxido-dismutase, também sofrem influência dos hormônios femininos, principalmente do estrogênio, que eleva a ação da catalase ${ }^{(6,7)}$. Mondal e Raí (2002) relataram que o aumento do nível circulante de interleucina 1 (IL-1) produzida por macrófagos esplênicos pós-gonadectomia sugere ação inibitória dos hormônios gonadais na atividade citotóxica dos macrófagos(31).

Diante das possíveis interações descritas entre hormônios ovarianos e placentários e o sistema imunitário, o presente trabalho teve como objetivo verificar a influência da ooforectomia e da gravidez na função fagocitária do sistema mononuclear fagocitário.

\section{Material e método}

Este trabalho foi realizado de acordo com as recomendações das Normas Internacionais de Proteção aos Animais e do Código Brasileiro de Experimentação Animal (1988), e aprovado pela Comissão de Ética do Departamento de Cirurgia da Faculdade de Medicina da Universidade Federal de Minas Gerais (UFMG) ${ }^{(16,41)}$.

O presente estudo foi conduzido em 45 ratas wistar adultas, pesando $200 \pm 30 \mathrm{~g}$, divididas em três grupos $(n=15)$ :

- grupo 1 - controle;

- grupo 2 - ratas ooforectomizadas;

- grupo 3 - ratas grávidas. 
Todos os animais ficaram alocados em gaiolas próprias para ratos (cinco ratas/gaiola) e receberam água e ração à vontade. No grupo 2, sob anestesia inalatória com éter, realizou-se laparotomia mediana de três centímetros de extensão para a retirada dos ovários bilateralmente. O fechamento da parede abdominal foi em dois planos, com pontos simples, utilizando fio de polipropileno monofilamentar 5-0 para a aponeurose e mononáilon 4-0 para a pele. Para a indução de gravidez, as ratas do grupo 3 permaneceram cinco dias em gaiolas individuais com um macho sabidamente fértil e, posteriormente, elas foram reagrupadas em três gaiolas amplas, com cinco animais em cada uma.

Após um período de três meses pós-operatórios (grupos 2 e 3) e decorridos 15 dias após a separação do macho (grupo 4), todos os animais foram submetidos a avaliação da atividade fagocitária do SMF. Sob anestesia inalatória com éter sulfúrico, administrou-se a todos os grupos uma solução de enxofre coloidal marcada com ${ }^{99} \mathrm{mTc}$, e atividade de $3 \mathrm{mCi}(110 \mathrm{MBq})$, na dose de $1 \mathrm{ml} / \mathrm{kg}$ de peso, pela veia femoral. Decorridos trinta minutos, os animais foram mortos com dose excessiva de éter sulfúrico. Em seguida, através de toracolaparotomia mediana ampla, foram retirados segmentos, medindo um centímetro, do lobo hepático direito, da parte média do baço e do lobo inferior do pulmão esquerdo. Coletou-se também um coágulo sangüíneo obtido pela secção da veia cava caudal. Aproveitou-se ainda essa fase do experimento para confirmar a gravidez nas ratas do grupo 3 .

Os fragmentos de cada órgão e o coágulo sangüíneo foram pesados e colocados em copos plásticos de $30 \mathrm{ml}$. A radioatividade contida em cada amostra foi determinada colocando-se os copos com as amostras no centro do colimador de um cintilógrafo (Siemens, modelo Orbiter) para mensuração dos raios gama (gama-câmara). A radiação foi calculada por grama de tecido. Previamente, dois copos vazios foram colocados no colimador da gama-câmara para a certificação da completa ausência de atividade radioativa. Atribuiu-se à somatória da radioatividade emitida pelos quatro fragmentos o valor 100. Com base nessa soma, a radioatividade de cada amostra (fígado, baço, pulmão e coágulo sangüíneo) foi convertida para valores percentuais.

As médias das radiações das amostras de cada animal e dos diferentes grupos foram comparadas utilizando-se a análise de variância e o teste $t$ de Student. Foram consideradas significativas as diferenças correspondentes a $p$ $<0,05^{(5)}$.

Biópsias de fígado, baço e pulmão de todos os animais foram estudadas à microscopia ótica, com o objetivo de avaliar possíveis alterações na celularidade do SMF. As amostras haviam sido previamente fixadas em formol a $10 \%$ e processadas em lâminas com coloração por hematoxilina e eosina (HE).

\section{Resultados}

Os animais permaneceram sem anormalidades aparentes durante o período de acompanhamento. Todas as ratas do grupo 3 tiveram sua gravidez comprovada pela identificação de fetos em seus úteros.

A Tabela apresenta os valores percentuais dos registros de radioatividade nas amostras dos órgãos estudados, bem como no coágulo sangüíneo. A captação de enxofre coloidal em todos os grupos foi maior no fígado $(p<0,05)$, seguido por baço e pulmão. No coágulo sangüíneo encontrou-se apenas uma pequena quantidade remanescente de colóide radioativo. No grupo 2 não houve diferença de captação coloidal em comparação com o grupo controle. No grupo 3 , a captação hepática foi proporcionalmente menor do que nos demais grupos $(p<0,05)$. Em compensação, a captação pulmonar das ratas grávidas foi maior $(p<0,05)$.

\section{Valores percentuais (média \pm erro padráo da média) dos registros de radioatividade em amostras de}

Amostras

$\begin{array}{lc} & \text { Grupo } 1 \\ \text { Fígado } & 60,12 \pm 8,52 \\ \text { Baço } & 30,2 \pm 12,8 \\ \text { Pulmão } & 8,16 \pm 5,25 \\ \text { Coágulo } & 1,52 \pm 0,81\end{array}$

Radioatividade (\%/g)

$$
\text { Grupo } 2
$$

Grupo 3

$59,25 \pm 12,2$

$28,7 \pm 8,42$

$10,34 \pm 7,21$

$1,61 \pm 1,12$
$52,34 \pm 14,65^{*}$

$29,23 \pm 12,3$

$16,7 \pm 9,31^{* *}$

$1,73 \pm 0,85$

Crupo 1: controle; grupo 2: ratas ooforectomizadas; grupo 3: ratas grávidas.

*Diminuição da captação hepática no grupo 3 em comparação com os demais $(p<0,05)$; **aumento da captação pulmonar no grupo 3 em comparação com os demais $(p<0,05)$. 
Alterações na histoarquitetura dos órgãos estudados não foram observadas. Afecções inflamatórias, proliferativas ou degenerativas também não foram constatadas. A celularidade hepática, esplênica e pulmonar também não foi diferente entre as ratas dos três grupos.

\section{Discussão}

A avaliação da função do SMF mediante a captação celular de enxofre coloidal marcado com ${ }^{99} \mathrm{mTc}$ vem sendo estudada numa linha de pesquisa desde $1981^{(4,12,27,28,37-40,42,}$ 43,45). A eficácia dessa captação celular por fagocitose de um colóide marcado, reconhecido como antígeno pelas células do SMF, é medida por um aparelho de gama-câmara que quantifica a radioatividade emitida por pequenas amostras, sendo proporcional ao número de partículas fagocitadas. No presente trabalho, aguardaram-se trinta minutos após a inoculação intravenosa de enxofre coloidal marcado com ${ }^{99} \mathrm{mTc}$, para permitir que essa substância fosse totalmente removida da circulação sangüínea pelo SMF, mesmo sabendo-se que $90 \%$ da depuração sangüínea ocorre nos primeiros cinco minutos ${ }^{(4,12)}$.

Para a análise da função do SMF, avaliou-se a captação do colóide apenas em fígado, pulmão e baço, por serem os principais órgãos responsáveis pela depuração sangüínea ${ }^{(38-40)}$. A mensuração da captação do enxofre coloidal nos demais órgãos do SMF, tais como placas de Peyer, medula óssea e linfonodos, não se procedeu por não ser, rotineiramente, factível $(4,38,40)$. Mediu-se também o colóide remanescente no sangue, com o objetivo de verificar a eficácia da função depuradora do SMF. A presença de valores muito reduzidos de colóide radioativo na circulação indicou que o SMF das ratas de todos os grupos desempenhou adequadamente o seu papel de remover partículas anômalas da circulação(28).

Para a marcação de diferentes estruturas biológicas, vários radiofármacos vêm sendo utilizados, como carbono, gálio, hidrogênio, rênio, tecnécio, entre outros ${ }^{(8,17,21)}$. O emprego do tecnécio foi devido as suas características fisicoquímicas e biológicas e por permitir um método de marcação simples, mas preciso, efetivo, rápido, de fácil avaliação e de baixo custo. Outra alternativa para se estudar a função do SMF é a depuração de bactérias também marcadas com ${ }^{99} \mathrm{mTc}$, mas este não foi o objetivo deste trabalho ${ }^{(17,27,28,45)}$.

A utilização de rata wistar é bastante comum em estudos experimentais que abordam gravidez devido a sua destacada característica biológica previamente conhecida: facilidade para cópula e período gestacional relativamente curto, em torno de 21 dias $^{(3,29)}$. Outros aspectos importantes são o pequeno porte, a grande resistência a procedimentos cirúrgicos, além da facilidade de manuseio e confinamento $(27,28,38,50)$.

A maior captação do radiofármaco pelo fígado foi esperada para todos os grupos, tendo-se em vista o grande fluxo sangüíneo hepático e a maior quantidade de componentes do SMF, representada principalmente pelas células de Kupffer, que são responsáveis por até $60 \%$ da capacidade fagocitária do organismo ${ }^{(37-39)}$. A redução da depuração hepática em presença de gravidez não pode ser explicada apenas com base nos achados do presente trabalho. Tindall e Beazley (1965), estudando as funções do fígado na gravidez, encontraram relação entre o elevado nível de estrogênio circulante e as alterações funcionais desse órgão ${ }^{(51)}$. Neste experimento não foram dosados os hormônios circulantes por dificuldade metodológica, tendo-se em vista a grande quantidade de sangue necessária para a formação do coágulo e por não ter sido objetivo do estudo a avaliação endócrina. Hiperbilirrubinemia, desglicogenização, aumento do teor de aminoácidos e hepatomegalia também já foram descritos durante o período gestacional( ${ }^{(11,46)}$.

O baço é responsável por $25 \%$ a $30 \%$ da depuração sangüínea, seguido pelos pulmões, com $5 \%$ a $10 \%$ dessa função ${ }^{(18,28,46)}$. Neste trabalho não foi verificada mudança na captação do colóide pelo baço provocada pela ooforectomia ou gravidez, apesar de alguns autores terem encontrado interação entre os hormônios sexuais no eixo hipotalâmico-hipófisário-gonadal-tímico ${ }^{(15,25,35)}$. Entretanto houve aumento da depuração pulmonar nas ratas grávidas. Sabe-se que durante o período gestacional ocorre elevação dos níveis de progesterona, responsável pelo aumento do volume corrente e pela freqüência respiratória ${ }^{(10)}$. A hiperventilação, com conseqüente aumento da pressão parcial de oxigênio $\left(\mathrm{PaO}_{2}\right)$ e diminuição da pressão parcial de gás carbônico $\left(\mathrm{PaCO}_{2}\right)$, é outro fator que parece influenciar o sistema de defesa celular ${ }^{(30)}$.

Embora a gravidez não seja uma condição descrita como predisponente para sepse, observou-se aumento da mortalidade de gestantes por influenza ${ }^{(33)}$. É provável que níveis elevados de progesterona e de 17-beta estradiol propiciem aumento da incidência de infecções fúngicas pulmonares ${ }^{(13,14)}$.

Alterações morfológicas nos órgãos estudados também não foram observadas. Todavia Oner et al. (2002), ao pesquisarem os efeitos de hormônios gonadais na histoarquitetura do timo, verificaram aumento de seu peso em presença de gonadectomia. Após a reposição hormonal, 
houve redução desse órgão e elevação do número de macrófagos e mastócitos ${ }^{(34)}$.

\section{Conclusões}

Com base nos dados do presente trabalho, conclui-se que a atividade fagocitária do sistema mononuclear fagocitário não se alterou em presença de ooforectomia, porém, em presença de gravidez murina, houve diminuição da fagocitose no fígado e aumento da função depuradora pulmonar.

\section{Agradecimentos}

Os autores agradecem ao Sr. Darcy Ferreira dos Santos pela gentil concessão dos animais utilizados nesta pesquisa e ao Prof. Carlos Jorge Rodrigues Simal pela realização dos exames cintilográficos. Somos gratos também ao Conselho Nacional de Desenvolvimento Científico e Tecnológico (CNPq) e à Fundação de Amparo à Pesquisa do Estado de Minas Gerais (FAPEMIG) pelos auxílios financeiros que permitiram a realização deste trabalho.

\section{Referências}

I. AHMED, A. S. et al. Gender and risk of autoimmune diseases. Environ Health Perspect, 107: 681-4, 1999.

2.AHMED,A. S.; PENHALE,W. J:; TALAL, N. Sex hormones, immune responses, and autoimmune diseases. Am J Pathol, | 2 1: $53|-5|, 1985$.

3. ALBRECHT, E. D. Pregnancy in young and aged rats: peripheral serum progesterone concentrations. Biol Reprod, 33: 432-5, 1985.

4. ALMEIDA, L. M. et al. Efeito da heparina sobre o sistema mononuclear fagocitário. Acta Cir Bras, I 1: 15-6, 1996.

5. ALTMAN, D. G.; GARDNER, M. J. Means and their differences. In: ALTMAN, D. G. et al. Statistics with confidence. Br Med J Books, 2000

6. AZEVEDO, R. B. et al. Gonadectomy impairs lymphocyte proliferation and macrophage function in male and female rats. Cell Biochem Funct, 15: 293-8, 1997.

7.AZEVEDO, R. B. et al. Regulation of antioxidant enzyme activities in male and female rat macrophages by sex steroids. Braz J Med Biol Res, 34: 683-7, 2001

8. BERNARDO, M. F.; PEREIRA, J. A. A.; BOASQUEVISQUE, E. M. Labeling of Klebsiella pneumoniae with technetium-99m. Rev Microbiol, 17: I 88-93, 1986.

9. BJORKSTEN, B. et al. Polymorphonuclear leucocyte function during pregnancy. Scan Immunol, 8: 257-62, 1978.

10. BROWN, E. J.; HOSEA, S. W.; FRANK, M. M. The role of complement in the localization of pneumococci in the splanchnic reticuloendothelial system during experimental bacteremia.J Immunol, 126: 2230-5, 1981.

I I. COMBES, B.; ADAMS, R. H. Pathophysiology of the liver in pregnancy. In: ASSALI, N. S. Pathophysiology of gestation. N.Y.: Academic Press, 1971.

12. DUVAL, I. A. et al.Tc sulphur colloid uptake by rats liver, spleen and lungs in early biliary obstruction. Med Science, 24: 2456, 1996.

13. FINLAND, M.; DUBLIN, T. D. Pneumococcus pneumoniae complicating pregnancy and the puerperium. JAMA, I12: 1027-32, 1939.

14. GARLAND, S. M. et al. Mechanisms, organisms and markers of infection in pregnancy.J Reprod Immunol, 57: I 69-83, 2002.

15. GROSSMAN, C. J. Interactions between the gonadal steroids and the immune system. Science, 227: 257-6I, 1985.
16. HOFF, C. Immoral and moral uses of animals. N Engl J Med, 302: II5-8, 1980.

17. HOLDSWORTH, R. J. et al. Blood clearance and tissue distribution of ${ }^{99} \mathrm{Tc}$-labelled pneumococci following splenectomy in rabbits. BrJ Exp Pathol, 70: 669-77, 1989.

18. HOSEA, S. W. et al. Opsonic requirements for intracellular clearance after splenectomy. N Engl J Med, 304: 245-50, 1981.

19. JENSEN, E.; WOOD, C.; KELLER, W. M. The normal increase in adrenal secretion during pregnancy contributes to maternal volume expansion and fetal homeostasis. J Soc Gynecol Investig, 9: 362-7I, 2002

20. JOHNSTON, R. B. Current concepts: immunology, monocytes and macrophages. N Engl J Med, 3 I 8: 747-52, 1988.

21. KATZ, S. et al. Blood clearance and organ localization of Candida albicans and E. coli following dual infection in rats. J Pediatr Surg, 28: 329-33, 1993.

22. KLEIN, H. H.; PICH, S. Cardiovascular changes during pregnancy. Herz, 28: 173-4, 2003

23. KNOFERL, M. W. et al. Female sex hormones regulate macrophage function after trauma-hemorrhage and prevent increased death rate from subsequent sepsis. Ann Surg, 235: 105-12, 2002.

24. KRAUSE, P. J. et al. Host defense during pregnancy. Am J Obstet Gynecol, 157: 274-80, 1987.

25. LACAVA, Z. G.; LUNA, H. The anticlastogenic effect of tocopherol in peritoneal macrophages of benznidazoletreated and ovariectomized mice. Mutat Res, 305: | 45-50, 1994.

26. MALTZER, M. C.; SILVA, J. JR. In vitro defects of phagocyte chemotaxis during pregnancy. I Clin Microbiol, I1: 170-3, 1980.

27. MARQUES, R. G. et al. Bacterial clearance after total splenectomy and splenic autotransplantation in rats. Applied Radiation and Isotopes, 57: 767-7I, 2002.

28. MARQUES, R. G. et al. Importância da preservação de tecido esplênico para a fagocitose bacteriana. Acta Cir Bras, 17: 388-93, 2002.

29. MATTHEIJ, J. A.; SWARTS, J. J. Quantification and classification of pregnancy wastage in 5-day cyclic young through middleaged rats. Lab Anim, 25: 30-4, 1991. 
30. MILNE, J. S.; HOWIE, A. D.; PACK, A. I. Dyspnoea during normal pregnancy. BrJ Obstet Gynaecol, 85: 260-3, 1978.

31. MONDAL, S.; RAI, U. In vitro effect of sex steroids on cytotoxic activity of splenic macrophages in wall lizard. Gen Comp Endocrinol, 125: 264-7I, 2002.

32. NEWSHOLME, P. et al. The importance of fuel metabolism to macrophage function. Cell Biochem Funct, 14: 1 - 10, 1996.

33. OKONTA, P. I. et al. Exploring the causes of and risk factors for maternal deaths in a rural Nigerian referral hospital.J Obstet Gynaecol, 22: 626-9, 2002.

34. ONER, H.; OZAN, E. Effects of gonadal hormones on thymus gland after bilateral ovariectomy and orchidectomy in rats. Arch Androl, 48: I I 5-26, 2002.

35. PAJOVIC, S. B. et al. Effects of progesterone and estradiol benzoate on glutathione dependent antioxidant enzyme activities in the brain of female rats. Gen Physiol and Biophysics, 18: 35-44, 1999.

36. PATTON, P.E.; COULAM, C. B.; BERGSTRALH, E.The prevalence of autoantibodies in pregnant and nonpregnant women. Am J Obstet Gynecol, I 57: 1 345-50, 1987.

37.PETROIANU,A.Avaliação da função fagocitária em remanescentes de esplenectomia subtotal e auto-implante esplênico autógeno. Rev Bras Hematol Hemoter, 25: 25-30, 2003.

38. PETROIANU,A.; SIMAL, C. J. R. Shifts in the reticuloendothelial system uptake patter induced by carbon colloid in the rats. Med Sci Res, 21 : 31 I-2, 1993.

39. PETROIANU, A.; SIMAL, C. J. R.; BARBOSA, A. J. A. Assessment of phagocytic function in remnants of subtotal splenectomy and autologous spleen implantation. Med Sci Res, 22: 7I 5-7, 1993.

40. PETROIANU, A.; SIMAL, C. J. R.; BARBOSA, A. J. A. Impairment of phagocytosis by mammalian splenic magrophages by $99 \mathrm{~m}$ Tc sulphur colloid. Med Sci Res, 20: 874-9, 1992.

4I. PETROIANU, A. Pesquisa experimental. In: PETROIANU, A.
Ética, moral e deontologia médicas. I. ed. Rio de Janeiro: Ganabara Koogan; 2000. p. 185-90.

42. RESENDE, V.; PETROIANU, A. Funções do remanescente esplênico após esplenectomia subtotal para o tratamento de lesões complexas do baço humano. Rev Assoc Med Bras, 48: 26-31, 2002.

43. RESENDE, V.; PETROIANU, A. Late functional study of human spleen autotransplantation after severe splenic injuries. Rev Col Bras Cir, 28: 165-70, 2001.

44. SABA, T. M. Physiology and physiopathology of the reticuloendothelial system. Arch Intern Med, 126: 1031-52, 1970.

45. SILVA, R. G. et al. Influência das operações sobre o baço na distribuição da Escherichia coli no sistema mononuclear fagocitário. Rev Col Bras Cir, 30: 65-7I, 2003.

46. SONG, C. S. et al. Hormones and the liver. Am J Obstet Gynecol, 105: 8|3-47, 1969.

46. SOUZA, A. I.; MALAQUIAS, B.; FERREIRA, L. O. C. Alterações hematológicas e gravidez. Rev Bras Hematol Hemoter, 24: 29-36, 2002.

47. SPEROFF, T. et al. A risk-benefit analysis of elective bilateral oophorectomy. Am J Obstet Gynecol, 164: |65-74, I991.

48. STERNBERG, E. M. Neuroendocrine regulation of autoimmune/ inflammatory disease.J Endocrinol, 169: 429-35, 2001.

49. TAKEUCHI, A.; PERSELLIN, R. H. The inhibitory effect of pregnancy serum on polymorphonuclear leukocyte chemotaxis. J Clin Lab Immunol, 3: 121-4, 1980.

50. INDALL, V. R.; BEAZLEY, J. M. An assessment of changes in liver function during normal pregnancy: using a modified bromsulphthalein test. J Obstet Gynaecol Br Commonw, 72: 717-37, 1965.

5।.WEINBERG, J. B. Mononuclear phagocytes. In: LEE, G. R. et al. Wintrobe's Clinical Hematology. I O. ed. Baltimore:Williams \& Wilkins; 1999. p. 337-9. 
O arquivo disponível sofreu correções conforme ERRATA publicada no Volume 41 Número 5 da revista. 